\title{
INDEX TO VOLUME 84
}

\author{
INVITED ADDRESSES
}

Anderson, D. W., Fibrations and geometric realizations, 765

Birtel, Frank T., Holomorphic approximation to boundary value algebras, 406

Cannon, J. W., The recognition problem: what is a topological manifold?, 832

Daverman, Robert J., Embeddings of $(n-1)$-spheres in Euclidean n-space, 377

Eisenbud, David, An alge braic approach to the topological degree of a smooth map, 751

Evans, Trevor, Word problems, 789

Federer, Herbert, Colloquium lectures on geometric measure theory, 291

Fife, Paul C., Asymptotic states for equations of reaction and diffusion, 693

Fröhlich, J., The pure phases (harmonic functions) of generalized processes or: Mathematical physics of phase transitions and symmetry breaking, 165

Goldschmidt, Hubert, The integrability problem for Lie equations, 531

Greenberg, J. M., Hassard, B. D. and Hastings, S. P., Pattern formation and periodic structures in systems modeled by reaction-diffusion equations, 1296

Hajian, Arshag and Ito, Yuji, Transformations that do not accept a finite invariant measure, 417

Hassard, B. D. See Greenberg, J. M.

Hastings, S. P. See Greenberg, J. M.

Hejhal, Dennis A., Monodromy groups and Poincaré series, 339

Hochster, Melvin, Some applications of the Frobenius in characteristic 0, 886

Hunt, Richard A., Harmonic measure and estimates of Green's function, 194

Ito, Yuji. See Hajian, Arshag

Kamber, Franz W. and Tondeur, Philippe, G-foliations and their characteristic classes, 1086

Keller, Joseph B., Rays, waves and asymptotics, 727

Kinderlehrer, David, Variational inequalities and free boundary problems, 7

Lashof, R., Equivariant smoothing theory, 1

Liulevicius, Arunas, Homotopy rigidity of linear actions: characters tell all, 213

Marsden, Jerrold E., Qualitative methods in bifurcation theory, 1125

McCrimmon, Kevin, Jordan algebras and their applications, 612

McKean, H. P. and Trubowitz, E., Hill's surfaces and their theta functions, 1042

Mitchell, Barry, Some applications of module theory to functor categories, 867

Montgomery, Hugh L., The analytic principle of the large sieve, 547

Niederreiter, Harald, Quasi-Monte Carlo methods and pseudo-random numbers, 957

Osserman, Robert, The isoperimetric inequality, 1182

Rosenthal, Haskell P., Some recent discoveries in the isomorphic theory of Banach spaces, 803

Siu, Yum-Tong, Pseudoconvexity and the problem of Levi, 481

Smith, Kennan T., Solmon, Donald C. and Wagner, Sheldon, L., Addendum to "Practical and mathematical aspects of the problem of reconstructing objects from radiographs", 691

Soare, Robert I., Recursively enumerable sets and degrees, 1149

Solmon, D. C. See Smith K. T.

Stasheff, James D., Continuous cohomology of groups and classifying spaces, 513

Stein, Elias M. and Wainger, Stephen, Problems in harmonic analysis related to curvature, 1239

Stein, Michael R., Whitehead groups of finite groups, 201

Taylor, Jean E., Crystalline variational problems, 568

Taylor, Michael E., Propagation, reflection, and diffraction of singularities of solutions to wave equations, 589

Tondeur, Philippe. See Kamber, Franz W.

Trubowitz, E. See McKean, H. P. 
Wagner, S. L. See Smith, K. T.

Wainger, Stephen. See Stein, Elias M.

\section{BOOK REVIEWS}

Alexander, Stephanie, A comprehensive introduction to differential geometry by Michael Spivak, 27

Alperin, J. L., Modular representations of finite groups by B. M. Puttaswamaiah and John D. Dixon, 32

Arkowitz, Martin, Hopf spaces by Alexander Zabrodsky, 929

Arrow, Kenneth J., Theory of optimal search by Lawrence D. Stone, 649

Axler, Sheldon, Brownian motion, Hardy spaces and bounded mean oscillation by K. E. Petersen, 645

Ayoub, R. G., Trigonometrical sums in number theory by I. M. Vinogradov, 250

Baker, Alan. See Stolarsky, Kenneth B.

Balakrishnan, A. V. See Holmes, Richard B.

Barbu, Viorel. See Crandall, Michael G.

Bellman, R. E., Combinatorial optimization: networks and matroids by Eugene L. Lawler, 461

Bennett, Colin, Interpolation spaces, and introduction by Jöran Bergh and Jörgen Löfström, 110

Bergh, Jöran. See Bennett, Colin.

Berlekamp, E. R., The theory of information and coding: A mathematical framework for communication by Robert $\mathrm{J}$. McEliece, 1351

Bishop, Yvonne M. M. See Nerlove, Marc

Blass, Andreas, Applied nonstandard analysis by Martin Davis; Introduction to the theory of infinitesimals by K. D. Stroyan and W. A. J. Luxemburg; and Foundations of infinites. imal calculus by Jerome Keisler, 34

Boas, R. P., Completeness and basis properties of sets of special functions by J. R. Higgins, 642

Boas, R. P. and Thron, W. J., Applied and computational complex analysis. II by Peter Henrici, 943

Boltjanski, V. G. See Clarke, Frank H.

Bona, Jerry, Differential equations and their applications by M. Braun, 41

Bosanquet, R. G. See Kreisel, G.

Braun, M. See Bona, Jerry

Brezin, Jonathan. See Howe, Roger

Brillinger, David R., Doubly stochastic Poisson processes by Jan Grandell, 463

Burks, Arthur W., The new elements of mathematics by Charles S. Peirce, 913

Cameron, Peter J. See Kantor, W. M.

Castaing, C. See Ekeland, I.

Caubet, Jean-Pierre. See Nelson, Edward

Chacon, R. V. Introduction to ergodic theory by Ya. G. Sinai, 656

Chandrasekhar, S. and Swerdlow, N., A history of ancient mathematical astronomy by $O$. Neugebauer, 428

Chandler, Richard E. See Steiner, Anne K.

Christenson, Charles O. See Rudin, M. E.

Clarke, Frank H., Optimization, a theory of necessary conditions by L. W. Neustadt; Optimale steuerung diskreter Systems by V. G. Boltjanski; and The qualitative theory of optimal processes by R. Gabasov and F. Kirillova, 47

Cohn, P. M., Grundzüge der universellen Algebra by Herbert Lugowski, 124

Coifman, R. R. and Weiss, Guido, Littlewood-Paley and multiplier theory by R. E. Edwards and G. I. Gaudry, 242

Conrad, Paul, Orderable groups by Roberta Botto Mura and Akbar Rhemtulla, 647

Conway, J. H. See Fraenkel, A. S.

Corwin, Lawrence, Lie groups and compact groups by John F. Price, 663

Coxeter, H. S. M., Three-dimensional nets and polyhedra by A. F. Wells, 466

Crandall, Michael G., Nonlinear semigroups and differential equations in Banach spaces, by Viorel Barbu, 632 
Davis, Martin. See Blass, Andreas

Deimling, Klaus. See Redheffer, R. M.

Diestel, J. See Retherford, J. R.

Dilworth, R. P., Matroid theory by D. J. A. Welsh, 1353

Dixon, John D. See Alperin, J. L.

Douglis, Avron, The wave equation on a curved space-time by F. G. Friedlander, 222

Dym, H. See Sarason, Donald

Edwards, R. E. See Coifman, R. R.

Ekeland, I., Convex analysis and measurable multifunctions by C. Castaing and M. Valadier, 950

Fienberg, Stephen E. See Nerlove, Marc and Press, S. James

Flanders, Harley, Problems and theorems in analysis by G. Pólya and G. Szegö, 53

Fraenkel, A. S. On numbers and games by J. H. Conway and Surreal numbers by D. E. Knuth, 1328

Friedlander, F. G. See Douglis, Avron.

Gabasov, R. See Clarke, Frank H.

Gale, David, Mathematical theory of economic dynamics and equilibria by V. L. Makarov and A. M. Rubinov, 665

Garfinkel, R. S. See Johnson, Ellis

Gaudry, G. I. See Coifman, R. R.

Gaudry, Garth, Homogeneous Banach algebras by Hwai-Chiuan Wang, 446

Goldschmidt, David M., Examples of groups by Michael Weinstein, 444

Gonzalo, E. Reyes. See Monk, J. D.

Goodman, Roe W. See Koranyi, Adam

Grandell, Jan. See Brillinger, David R.

Grätzer, George A., Categories of algebraic systems by Mario Petrich and Mal'cev varieties by Jonathan D. H. Smith, 1339

Greenberg, Harold. See Johnson, Ellis

Greiner, P. C. See Rothschild, Linda Preiss

Gustafson, W. H., Linear representations of finite groups by Jean-Pierre Serre, 939

Hejhal, Dennis A. See Patterson, S. J.

Henrici, Peter. See Boas, R. P. and Thron, W. J.

Herstein, I. N. See Miers, C. R.

Higgins, J. R. See Boas, R. P.

Hochster, M., Finite free resolutions by D. G. Northcott, 652

Hoffmann, Christoph M., Recursive Funktionen in der Komputer Theorie by Rózsa Péter, 62

Hogbe-Nlend, Henri. See Waelbroeck, Lucien

Holland, Paul W. See Nerlove, Marc and Press, S. James

Holmes, Richard B., Applied functional analysis by A. V. Balakrishnan, 65

Howe, Roger, Harmonic analysis on compact solvmanifolds by Jonathan Brezin, 671

Howie, John M., Lectures in semigroups by M. Petrich, 453

Hu, T. C. See Johnson, Ellis

Iyanaga, S. See O'Meara, O. T.

James, Robert C., Geometry of spheres in normed spaces by Juan Jorge Schäffer, 71

Johnson, Ellis, Integer programming and network flows by T. C. Hu; Integer programming by Harold Greenberg; Integer programming by R. S. Garfinkel and G. L. Nemhauser; Mathematical programming by T. C. Hu and Stephen M. Robinson; Integer programming by Harvey M. Salkin; and Integer programming: Theory applications and computations by Hamdy A. Taha, 228

Joseph, Daniel D. See Rosenblat, S.

Kantor, W. M., Parallelisms of complete designs by Peter J. Cameron, 451

Kasch, Friedrich. See Reiten, Idun

Keisler, Jerome. See Blass, Andreas

Kirillova, F. See Clarke, Frank H.

Klamkin, M. S., Companion to concrete mathematics, Volumes I and II, by Z. A. Melzak, 114 
Knight, Frank B., Stochastic processes by John Lamperti, 677

Knuth, D. E. See Fraenkel, A. S.

Koranyi, Adam, Nilpotent Lie groups: Structure and applications to analysis by Roe W. Goodman, 674

Kreisel, G., Wittgenstein's lectures on the foundations of mathematics by R. G. Bosanquet, Norman Malcolm, Rush Rhees and Yorick Smythies, 79

Kunze, Ray A., The theory of unitary group representations by George W. Mackey, 73

Kushner, Harold J. See Papanicolaou, George C.

Kussmaul, A. U. See Protter, Philip

Lakshmikantham, V., Qualitative analysis of large scale dynamical systems, by Anthony $\mathrm{N}$. Michel and Richard K. Miller, 937

Lamperti, John. See Knight, Frank B.

Lawler, Eugene L. See Bellman, R. E.

Linnik, Ju. V. See Loève, Michel

Loève, Michel, Decomposition of random variables and vectors by $\mathrm{Ju}$. V. Linnik and I. V. Ostrovskií, 638

Löfström, Jörgen. See Bennett, Colin

Loos, Ottmar. See McCrimmon, Kevin

Lugowski, Herbert. See Cohn, P. M.

Luxemburg, W. A. J. See Blass, Andreas

Mackey, George W. See Kunze, Ray A.

MacWilliams, F. J. See Pless, Vera S.

Mahler, Kurt. See Stolarsky, Kenneth B.

Makarov, V. L. See Gale, David

Makkai, Michael. See Monk, J. D.

Malcolm, Norman. See Kreisel, G.

Martin, Robert H. Jr. See Redheffer, R. M.

Masser, David. See Moreno, Carlos.

McCrimmon, Kevin, Jordan pairs by Ottmar Loos, 685

McDonald, Bernard R. See Ohm, Jack

McEliece, Robert J. See Berlekamp, E. R.

McKean, H. P. See Sarason, Donald

Melzak, Z. A. See Klamkin, M. S.

Michel, Anthony N. See Lakshmikantham, V.

Miers, C. R., Rings with involution by I. N. Herstein, 231

Miller, Richard K. See Lakshmikantham, V.

Mitchell, Barry M., Theory of modules by Alexandru Solian, 1336

Monk, J. D., First order categorical logic by Michael Makkai and Gonzalo E. Reyes, 1378

Moreno, Carlos, Elliptic functions and transcendence by David Masser, 456

Mukherjea, Arunava. See Rosenblatt, Murray

Mura, Roberta Botto. See Conrad, Paul

Naber, Gregory L. See Rudin, M. E.

Nagell, Trygve. See Schmidt, Wolfgang M.

Nelson, Edward, Le mouvement brownien relativiste by Jean-Pierre Caubet, 121

Nemhauser, G. L. See Johnson, Ellis

Nerlove, Marc and Press, S. James, Discrete multivariate analysis: Theory and practice by Yvonne M. M. Bishop, Stephen E. Fienberg and Paul W. Holland, 470

Neuberger, J. W., Near-rings, the theory and its applications by Gunter Pilz, 934

Neugebauer, O. See Chandrasekhar, S. and Swerdlow, N.

Neustadt, L. W. See Clarke, Frank H.

Northcott, D. G. See Hochster, M.

Ohm, Jack, Geomteric algebra over local rings by Bernard R. McDonald, 75

O'Meara, O. T., The theory of numbers by S. Iyanaga, 90

Ostrovskiî, I. V. See Loève, Michel

Papanicolaou, George C., Probability methods for approximations in stochastic control and for elliptic equations by Harold J. Kushner, 262

Patterson, S. J., The Selberg trace formula for PSL(2, R), Volume I by Dennis A. Hejhal, 256 
Peirce, Charles S. See Burke, Arthur W.

Péter, Rózsa. See Hoffmann, Christoph M.

Petersen, K. E. See Axler, Sheldon.

Petrich, M. See Howie, John M.

Petrich, Mario. See Grätzer, George A.

Pilz, Gunter. See Neuberger, J. W.

Pless, Vera S., The theory of error-correcting codes. I and II by F. J. MacWilliams and N. J.

A. Sloane, 1356

Pólya, G. See Flanders, Harley

Press, S. James. See Nerlove, Marc

Price, John F. See Corwin, Lawrence

Protter, Philip, Stochastic integration and generalized martingales by A. U. Kussmaul, 1346

Puttaswamaiah, B. M. See Alperin, J. L.

Redheffer, R. M., Nonlinear operators and differential equations in Banach spaces by Robert H. Martin, Jr. and Ordinary differential equations in Banach spaces by Klaus Deimling, 628

Reid, Constance. See Stone, Marshall $\mathrm{H}$.

Reiten, Idun, Moduln und Ringe by Friedrich Kasch, 925

Retherford, J. R., Vector measures by J. Diestel and J. J. Uh1, Jr., 681

Reyes, Gonzalo E. See Monk, J. D.

Rhees, Rush. See Kreisel, G.

Rhemtulla, Akbar. See Conrad, Paul

Robbins, H. E. Adventures of a mathematician by S. M. Ulam, 107

Robinson, Stephen M. See Johnson, Ellis

Rosenblat, S., Stability of fluid motions. I, II by Daniel D. Joseph, 96

Rosenblatt, Murray, Measures on topological semigroups: Convolution products and random walks by Arunava Mukherjea and Nicholas A. Tserpes, 104

Rothschild, Linda Preiss, Estimates for the M. Stein, 266

Rubinov, A. M. See Gale, David

Rudin, M. E., Aspects of topology by Charles O. Christenson and William L. Voxman; and Set-theoretic topology: With emphasis on problems from the theory of coverings, zero dimensionality and cardinal invariants by Gregory L. Naber, 271

Rutishauser, Heinz. See Stewart, G. W.

Sachs, R. K. See Thorpe, John A.

Salkin, Harvey M. See Johnson, Ellis

Sarason, Donald, Gaussian processes, function theory, and the inverse spectral problem by

H. Dym and H. P. McKean, 260

Schäffer, Juan Jorge. See James, Robert C.

Schmidt, Wolfgang M., Selected mathematical papers of Axel Thue edited by Trygve Nagell, Atle Selberg, Sigmund Selberg and Knut Thalberg, 919

Selberg, Atle. See Schmidt, Wolfgang M.

Selberg, Sigmund. See Schmidt, Wolfgang $M$.

Serre, Jean-Pierre. See Gustafson, W, H.

Sinai, Ya. G. See Chacon, R. V.

Sloane, N. J. A. See Pless, Vera S.

Smale, Stephen, Catastrophe theory: Selected papers by E. C. Zeeman, 1360

Smith, Jonathan D. H. See Grätzer, George A.

Smythies, Yorick. See Kreisel, G.

Solian, Alexandru. See Mitchell, Barry M.

Spivak, Michael. See Alexander, Stephanie

Stein, E. M. See Rothschild, Linda Preiss

Steiner, Anne K., Hausdorff compactifications edited by Richard E. Chandler, 448, erratum, 1381

- Supercompactness and Wallman spaces by J. Van Mill, 918

Stewart, G. W., Vorlesungen über numerische Mathematik by Heinz Rutishauser, 660 
Stolarsky, Kenneth B., Transcendental number theory by Alan Baker; Lectures on transcendental numbers by Kurt Mahler; Nombres transcendants by Michel Waldschmidt, 1370

Stone, Lawrence D. See Arrow, Kenneth J.

Stone, Marshall H., Courant in Göttingen and New York-The story of an improbable mathematician by Constance Reid, 234

Stroyan, K. D. See Blass, Andreas

Swerdlow, Noel. See Chandrasekhar, S.

Szegö, G. See Flanders, Harley

Taha, Hamdy A. See Johnson, Ellis

Thalberg, Knut. See Schmidt, Wolfgang M.

Thorpe, John A., General relativity for mathematicians by R. K. Sachs and H. Wu, 1344

Thron, W. J. See Boas, R. P.

Tserpes, Nicholas A. See Rosenblatt, Murray

Uhl, J. J., Jr. See Retherford, J. R.

Ulam, S. M. See Robbins, H. E.

Valadier, M. See Ekeland, I.

Van Mill, J. See Steiner, Anne K.

Vinogradov, I. M. See Ayoub, R. G.

Voxman, William L. See Rudin, M. E.

Waelbroeck, Lucien, Bornologies and functional analysis by Henri Hogbe-Nlend, 1368

Waldschmidt, Michel. See Stolarsky, Kenneth B.

Wang, Hwai-Chiuan. See Gaudry, Garth

Weinstein, Michael. See Goldschmidt, David M.

Weiss, Guido. See Coifman, Ronald R.

Wells, A. F. See Coxeter, H. S. M.

Welsh, J. A. See Dilworth, R. P.

Wu, H. See Thorpe, John A.

Zabrodsky, Alexander. See Arkowtiz, Martin

Zeeman, E. C. See Smale, Stephen

\section{RESEARCH ANNOUNCEMENTS}

Aharoni, Israel and Lindenstrauss, Joram, Uniform equivalence between Banach spaces, 281 Asimov, Daniel, Average Gaussian curvature of leaves of foliations, 131

Becker, Eberhard, Hereditarily pythagorean fields, infinite Harrison-primes and sums of $2^{n}$ th powers, 278

Berger, Marc A. and Mizel, Victor J.,A Fubini theorem for iterated stochastic integrals, 159

Calderón, A. P., Calderón, C. P., Fabes, E., Jodiet, M. and Riviere, N. M., Applications of the Cauchy integral on Lipschitz curves, 287

Calderón, C. P. See Calderón, A. P.

Canfield, E. Rodney, On a problem of Rota, 164

Cohen, F. R. and Taylor, L. R., Configuration spaces: applications to Gelfand-Fuks cohomology, 134

van Douwen, Eric K., Existence and applications of remote points, 161

Drazin, Michael P., Natural structures on semigroups with involution, 139

Fabes, E. See Calderón, A. P.

Gohberg, I., Lerer, L. and Rodman, L., Factorization indices for matrix polynomials, 275

Hagedorn, George A., Asymptotic completeness for a class of four particle Schrödinger operators, 155

Handelman, David and Lawrence, John, Finite Rickart C*-algebras, 157

Ioffe, Alexander D., Representation theorems for multifunctions and analytic sets, 142

Jodiet, M. See Calderón, A. P.

Kechris, A. S. and Martin, D. A., On the theory of $\Pi_{3}^{1}$ sets of reals, 149

Lawrence, John. See Handelman, David

Lerer, L. See Gohberg, I.

Lindenstrauss, Joram. See Aharoni, Israel

Martin, D. A. See Kechris, A. S.

Metropolis, N. and Rota, Gian-Carlo, On the lattice of faces of the n-cube, 284 
Mizel, Victor J. See Berger, Marc A.

Niederreiter, Harald, The serial test for linear congruential pseudo-random numbers, 273

Riviere, N. M. See Calderón, A. P.

Rodman, L. See Gohberg, I.

Rota, Gian-Carlo. See Metropolis, N.

Sigal, I. M., On quantum mechanics of many-body systems with dilation-analytic potentials, 152

Stenger, Frank, Upper and lower estimates on the rate of convergence of approximations in $H_{p}, 145$

Taylor, L. R. See Cohen, F. R.

Walsh, John J., Infinite dimensional compacta containing no $n$-dimensional $(n \geqslant 1)$ subsets, 137

Weisfeiler, B., On Lie algebras of differential formal groups of Ritt, 127

Williams, R, E., Errata to " $A$ note on rings with a weak algorithm", 1381

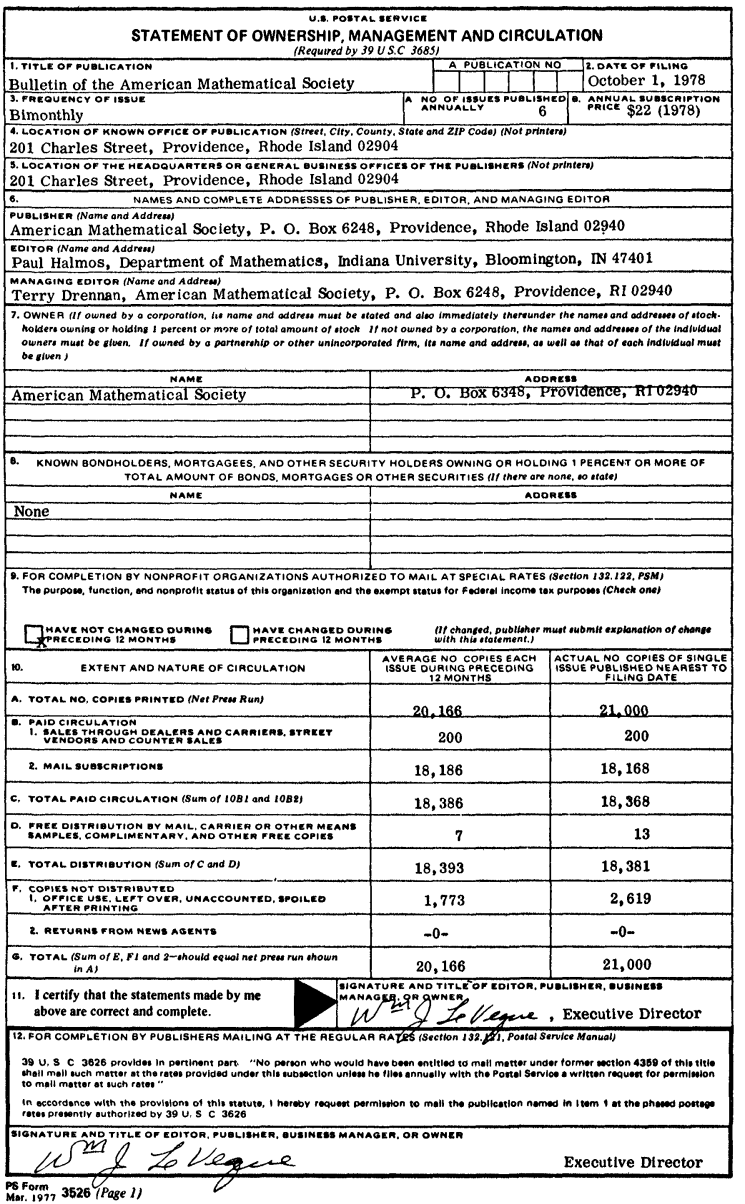

